\title{
Bladder Hernia, Bladder Stone and Contralateral Bowel Hernia: An Usual Combination
}

\author{
Akif Erbin1, Halil Lutfi Canat1, Burak Ucpinar1 and Hayati Malcik2
}

\begin{abstract}
We report and discuss a rare case of inguino-scrotal urinary bladder herniation $(\mathrm{IBH})$ complicated with a big urinary bladder stone and contralateral indirect inguinal bowel hernia. A 68-year male patient presented to the outpatient urology department with a 4-year history of right inguino-scrotal pain and swelling, which was gradually increasing in size, and of intermittent swelling in the left groin. Physical examination revealed right irreducible inguino-scrotal hernia and reducible left inguinal hernia extending to the groin. Computerised Tomography (CT) revealed that the right side of urinary bladder was herniating through the inguinal canal into the right hemiscrotum along with a $22 \mathrm{~mm}$ urinary bladder stone. The patient underwent bilateral Lichtenstein tension-free mesh hernioplasty through inguinal incisions and transurethral laser cystolithotripsy in the same session. To the best of the authors' knowledge, this is the first reported case of bilateral inguinal hernia with concomitant bladder stone in the literature.
\end{abstract}

Key Words: Bladder hernia, Bladder stone, Bowel hernia.

\section{INTRODUCTION}

Inguinal hernia is a common disorder which requires surgical repair. Many organs can be detected in the inguinal hernia sac, but bladder involvement (inguinoscrotal urinary bladder herniation; $\mathrm{IBH}$ ) is a rare condition which is encountered in $1-4 \%$ of all inguinal hernia cases. However, among obese men aged between 50 and 70 years, the frequency increases to about $10 \% .{ }^{1} \mathrm{IBH}$ complicated with bladder stone is an extremely rare condition; and only a few cases have been reported in the English-based literature.2

Herein, we report and discuss a case of IBH complicated with a big bladder stone and contralateral indirect inguinal bowel hernia. To the best of the authors' knowledge, this is the first reported case in the literature.

\section{CASE REPORT}

Written informed consent was obtained from the patient to report this case study. A68-year male, with a body mass index of $29 \mathrm{~kg} / \mathrm{m}^{2}$, presented to the outpatient urology department with a 4-year history of right inguino-scrotal pain and swelling, which was gradually increasing in size, and of intermittent swelling in the left groin. The patient also stated that he had a 2-year history of

1 Department of Urology, Haseki Training and Research Hospital, Istanbul, Turkey

2 Depatment of General Surgery, Dr. Munif Islamoglu State Hospital, Kastamonu, Turkey

Correspondence: Dr. Akif Erbin, Department of Urology, Haseki Training and Research Hospital, Istanbul, Turkey

E-mail: akiferbin@hotmail.com

Received: September 10, 2018; Accepted: February 22, 2019 moderate storage type lower urinary tract symptoms which were increasing intermittently. His past medical history included hypertension and hyper-lipidemia with no previous history of surgery. Physical examination revealed irreducible right inguino-scrotal hernia and reducible left inguinal hernia extending to the groin. Digital rectal examination revealed a slightly enlarged benign-feeling prostate. On uroflowmetry, maximum flow rate (Qmax) was $16.5 \mathrm{ml} / \mathrm{s}$ and the total voided volume was 187 cc (Figure 1). His PSA level was 2.1 $\mathrm{ng} / \mathrm{ml}$, serum creatinine $1.03 \mathrm{mg} / \mathrm{dL}$, and prostate volume was $30 \mathrm{cc}$ on transrectal ultrasonography. Urine culture demonstrated urinary tract infection and the patient was treated effectively with appropriate antibiotics. Due to the presence of microscopic hematuria in urinalysis, urine cytology was obtained and the result was negative for malignant cells. To rule out any anatomic abnormalities and concomitant pathologies, computed tomography (CT) was obtained and revealed that the right side of bladder was herniating through the inguinal canal into the right hemiscrotum and a $22 \mathrm{~mm}$ bladder stone was detected (Figures $2 a$ and $2 b$ ).

The patient underwent bilateral Lichtenstein tension-free mesh hernioplasty through inguinal incisions and transurethral laser cystolithotripsy in the same session. These procedures were performed in supine and lithotomy positions, under spinal anesthesia. The herniated bladder sac was dissected from the structures within the inguinal canal and the hemi-scrotum, then re-positioned to its original location within the pelvis (Figure 3). The inguinal floor was repaired using a polypropylene mesh. Similarly, Lichtenstein repair was performed with a mesh for the contralateral inguinal bowel hernia. Flexible cystoscope and Holmium:YAG laser were used to perform cystolithotripsy. Holmium: YAG laser settings 
were 0.8 Joule and 12 Hertz to achieve effective dusting. Milimetric particles were washed out to achieve complete clearance. Total operation time was 110 minutes (bilateral hernioplasty: 80 minutes and cystolithotripsy: 30 minutes). The post-operative recovery period was uneventful. Post-operative cystography was performed on $10^{\text {th }}$ post-operative day and did not reveal any extravasation. Thus, urethral catheter was removed. Patient was started on alpha blocker treatment (Tamsulosin, 0.4 $\mathrm{mg}$, once daily) after catheter removal. International prostate symptom score (IPSS) score was 6 and uroflowmetry revealed a Qmax value of $18 \mathrm{ml} / \mathrm{s}$, on 1st month follow-up.

\section{DISCUSSION}

Inguino-scrotal urinary bladder herniation is a very rare pathology and only 74 cases have been reported in the

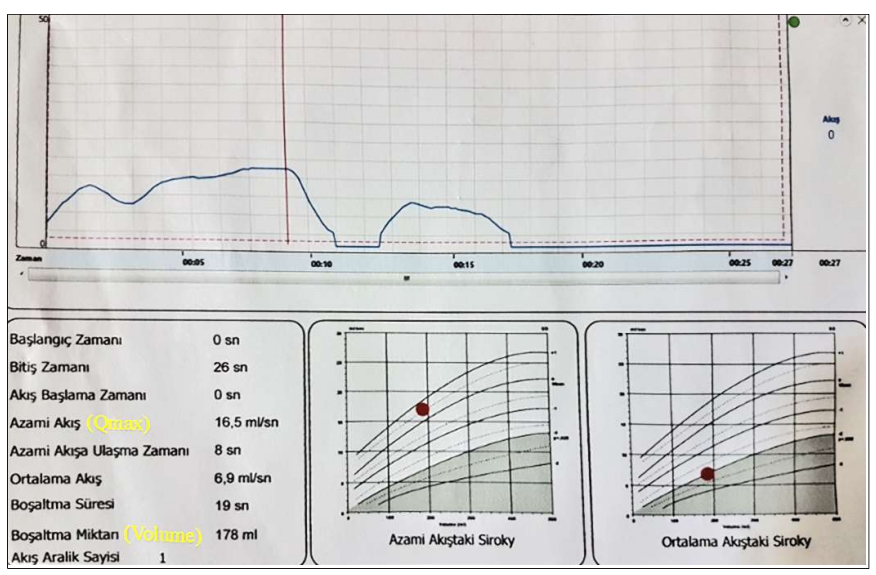

Figure 1: Uroflowmetry (Qmax was $16.5 \mathrm{ml} / \mathrm{s}$ and total voided volume was $178 \mathrm{~mL})$.

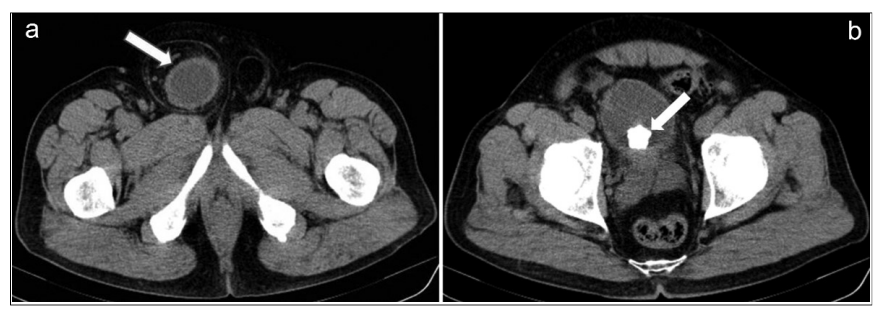

Figure 2: (a) Non-contrast CT axial view demonstrates herniation of bladder into the right scrotum (arrow). (b) Non-contrast CT shows big bladder stone (arrow)

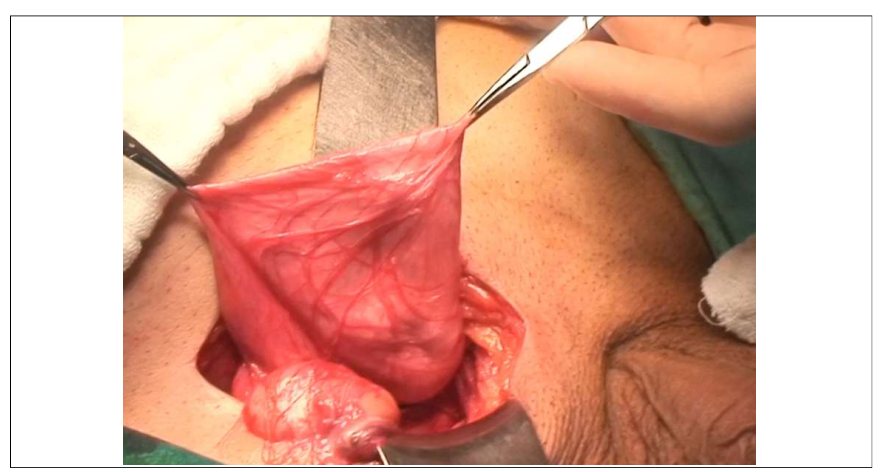

Figure 3: Intraoperative appearence of the herniated portion of the bladder (extraperitoneal bladder herniation). literature, in the last 10 years. ${ }^{3}$ Most IBH cases are asymptomatic and usually diagnosed incidentally during radiological evaluation or at the time of the surgery due to the small intermittent nature of these hernias. ${ }^{4}$ The preoperative identification of $\mathrm{IBH}$ can prevent iatrogenic bladder injuries and modify operative management of the inguinal hernia. IBH can be subdivided into three types (extraperitoneal, intraperitoneal, and paraperitoneal) according to their relationship with the parietal peritoneum. The paraperitoneal type, in which the extraperitoneal portion of the bladder lies along the medial wall of the sac, is the most common type of IBH. The present case can be classified as the extraperitoneal type, because the bladder was herniated directly into the inguinal canal without any relationship with the peritoneum. Pathophysiology of IBH might be related to the presence of bladder outlet obstruction (e.g. due to benign prostatic hypertrophy (BPH), obesity, chronically over distended bladder, prior surgeries or local trauma and weakness of supporting structures in the pelvic region. 5 The presence of bilateral hernia suggested the weakness of supporting structures as an etiological factor. The presence of mild BPH related symptoms and the achievement of relief with alpha-blocker treatment in this patient supported this causation.

Complications of IBH include urinary tract infection, sepsis, obstructive uropathology, vesicoureteral reflux, renal failure, strangulation of bladder wall, bladder rupture, and bladder stones.1,4,5 Oruc and colleagues reported that $11.2 \%$ of 190 cases of inguinal hernias were associated with urological findings such as herniation of bladder, ureter, and diverticulum. They also found that $11.2 \%$ of these hernias were associated with urological malignancies and $23.5 \%$ of these were associated with a variety of complications. ${ }^{4}$ These complications can be fatal, so, it is very important to confirm the IBH diagnosis at an early stage. The present case was complicated with a big bladder stone. Also, the patient had contralateral inguinal bowel hernia. This point was the distinctive feature of our case among other cases in the literature. However, due to the aforementioned underlying factors, bilateral hernia can also be encountered, and should be kept in mind in such patients.

In patients undergoing inguinal hernia repair, only less than $7 \%$ of IBH cases are diagnosed preoperatively. Thus, it is important to maintain a high index of suspicion in high-risk patients with inguinal hernias. High-risk groups include obese men with urologic symptoms, men with prior inguinal hernia repairs, and men older than 50 years. In these patient populations, some authors recommend performing a CT scan before inguinal hernia repair to evaluate the contents of the hernia. ${ }^{6}$ Standard treatment of bladder and bowel hernia is either reduction or resection of herniated bladder or bowel, followed by herniorraphy. The Lichtenstein tension-free mesh 
hernioplasty technique seems to be best option in most patients. In IBH surgery, bladder resection should be performed only for cases with a bladder wall necrosis, a tight hernial neck, or presence of a tumor in the herniated bladder sac. 7,8 If the patient has inguinal bladder hernia, inguinal bowel hernia and bladder stone, these three different pathologies can be managed in a single session.

In conclusion, it is important that urologists and general surgeons should be aware of the IBS diagnosis, and its complications and coexistent pathologies. Preoperative identification of $\mathrm{IBH}$ is crucial to prevent iatrogenic injuries or even more severe complications.

\section{REFERENCES}

1. Bisharat $M$, O'Donnell ME, Thompson $T$, MacKenzie $N$, Kirkpatrick D, Spence RA, et al. Complications of inguinoscrotal bladder hernias: A case series. Hernia 2009; 13:81-4.
2. Ng AC, Leung AK, Robson WL. Urinary bladder calculi in a sliding vesical-inguinal-scrotal hernia diagnosed preoperatively by plain abdominal radiography. Adv Ther 2007; 24:1016-9.

3. Branchu B, Renard Y, Larre S, Leon P. Diagnosis and treatment of inguinal hernia of the bladder: A systematic review of the past 10 years. Turk $J$ Urol 2018; 44:384-8.

4. Oruc MT, Akbulut Z, Ozozan O, Coskun F. Urological findings in inguinal hernias: A case report and review of the literature. Hernia 2004; 8:76-9.

5. Safavy S, Mitsinikos E, Tropea B, Chang A, Patel H. Obstructive uropathy and sepsis caused by an inguinoscrotal bladder hernia: A case report. Perm J 2018; 22:17-52.

6. Moufid K, Touiti D, Mohamed L. Inguinal bladder hernia: Four case analyses. Rev Urol 2013; 15:32-6.

7. Gomella LG, Spires SM, Burton JM, Ram MD, Flanigan RC. The surgical implications of herniation of the urinary bladder. Arch Surg 1985; 120:964-7.

8. Gurer A, Ozdogan M, Ozlem N, Yildirim A, Kulacoglu H, Aydin R. Uncommon contents in groin hernia sac. Hernia 2006; 10:152-5. 